\title{
Interacciones de medicamentos y eventos adversos en fármacos utilizados en una unidad de cuidados intensivos
}

\author{
JULIO PLAZA ${ }^{\text {la }}$, MAURICIO ÁLAMO ${ }^{2}$, PAULINA TORRES ${ }^{1 \mathrm{a}}$, \\ ÁLVARO FUENTES ${ }^{3 a}$, FERNANDO LÓPEZ ${ }^{1 a}$
}

'Departamento de Farmacia, Facultad de Química, Pontificia Universidad Católica de Chile. ${ }^{2}$ Unidad de Cuidados Intensivos (UCI) Clínica Dávila S.A. ${ }^{3}$ Servicio de Farmacia Clínica Dávila S.A.

aQuímico-Farmacéutico

Recibido el 16 de marzo de 2009, aceptado el 30 de marzo de 2010.

Correspondencia a: Julio Plaza D.

Av. Vicuña Mackenna 4860 Fax: 3544744 E-mail: jrplaza@uc.cl

Mauricio Álamo T. E-mail:malamo@davila.cl

\section{Drug interactions and adverse events induced by drugs used in an intensive care unit}

Background: Eleven percent of hospitalized patients experience drug-drug interactions (DDIs), elevating morbidity, mortality and health care costs. Polypharmacy is very common in intensive care units (ICUs), increasing the risks of drug adverse events (AEs). Aim: To assess DDIs in ICU patients. Material and Methods: A prospective study conducted in the ICU of a private hospital, evaluating the frequency of DDIs, AEs developed and their relationship. Patients admitted to the ICU were included if they stayed at least three days in the ICU and received at least one studied drug Results: Thirty five patients aged $59 \pm 16$ years (24 women) were enrolled in the study. Seventy six DDIs and 60 AEs were recorded. Statistically significant associations were only found for midazolam-fentanyl-propofol with bradycardia and hypotension and amphotericin B-vancomycin and vancomycin-amikacin with acute renal failure (ARF). Relative risks were 10.4 (95\% confidence intervals (CI) 1.59 - 68) for bradicardia, 5 (95\% CI 1.082 - 23.4) for hypotension and 6.4 (95\% CI 1.9 - 21.6) for ARF. The odds ratios were 125.2 (95\% CI 3 - 250), 12.6 (95\% CI 1.3 - 77) and 10.8 (95\% CI 1.3 - 282) respectively. Conclusions: DDIs associated with risk of AEs were fentanyl, propofol and midazolam for bradycardia and hypotension and amphotericin B-vancomycin and vancomycin-amikacin for ARF.

(Rev Med Chile 2010; 138: 452-460).

Key words: Drug interactions; Intensive care units; Polypharmacy.
L a interacción entre fármacos (IEF) se define como "la administración de dos o más fár$\checkmark$ macos con propiedades farmacocinéticas y farmacodinámicas particulares, con posibilidad de desencadenar eventos adversos (EA) posibles". Para la Organización Mundial de la Salud (OMS) los EA son todo efecto de un medicamento, que es perjudicial y no deseado, que ocurre a dosis usadas con fines terapéuticos, profilácticos o de diagnóstico ${ }^{1,2,4,5}$. El estudio de los EA y las IEF comenzó a realizarse en Chile el año 1995 con la creación de un programa nacional de farmacovigilancia, que buscaba entregar información sobre los medicamentos usados en el país y a la vez obtener casos de EA que pudieran ser parte de una futura base de notificaciones, que consta de 1.797 casos comunicados entre 1995 y 2001, con un peak para este último año de 771 notificaciones ${ }^{3,6}$. De los datos recibidos hasta el año 2001, los antimicrobianos ocupan el primer lugar con $31 \%$ de los reportes, seguidos por antipsicóticos, anticonvulsivantes y antiinflamatorios no esteroidales ${ }^{6}$.

En el caso de los Estados Unidos de Norteamérica el estudio de ambos factores determinó que producían un costo estimado de 76,6 billones de dólares para el año 1995, calculado en relación a la mortalidad y morbilidad por drogas ${ }^{7-9}$.

Se estima además, que cerca de $2,8 \%$ de las 
admisiones de los pacientes a los servicios de salud se debían a posibles IEF, que alrededor de $11 \%$ de los pacientes que estaban hospitalizados desarrolló algún síntoma relacionado con una IEF y que del total de pacientes que recibían medicamentos, $9,2 \%$ a $70,3 \%$ tuvo la posibilidad de desarrollar alguna IEF, que calculada según el costo anual fue equivalente a 130 billones de dólares en gastos para el servicio de salud. Finalmente, de todos los casos estudiados $1,2 \%$ a $23,3 \%$ de las IEF probabas presentaron relevancia clínica ${ }^{10-12}$.

Es importante que exista una vigilancia sobre las IEF relevantes que pudiesen desarrollar EA potencialmente peligrosos, asumir esta relación y determinar su incidencia. Esto permitirá adoptar las medidas que disminuyan la mortalidad y morbilidad de drogas asociados a ellos. Se destaca que los programas de acreditación de calidad instan a las instituciones de salud a incentivar la notificación e investigar cada caso que se produzca, coordinando la creación de nuevos departamentos o cargos que en la actualidad utilizan nuevas herramientas como los programas de screening de interacciones que apoyan la toma de una decisión (selección de medicamentos) ${ }^{13-18}$. El comité de farmacovigilancia de Clínica Dávila es encabezado por un químico-farmacéutico encargado desde el año 2007, quien reúne la información de los casos producidos en la institución y los informa a través de la ficha establecida por la CENIMEF (Centro Nacional de Información de Medicamentos y Farmacovigilancia) del Instituto de Salud Pública (ISP).

Creemos que las IEF y los EA son frecuentes en nuestros enfermos de unidad de cuidados intensivos (UCI), dada la polifarmacia habitual. Por otra parte, no evaluamos en forma rutinaria estos riesgos. El objetivo de nuestro trabajo es medir la frecuencia de las IEF y de los EA, para conocer la real importancia de cada uno de ellos dentro de nuestra UCI, destacando además que los trabajos actuales no poseen información sobre los pacientes UCI.

\section{Material y Métodos}

Se realizó un estudio prospectivo y de cohorte, en el cual se siguió los fármacos utilizados de los pacientes UCI entre los meses de junio y agosto del año 2008, evaluando las IEF y los EA de una lista de
14 distintas asociaciones de fármacos, clasificados como: 1) Contraindicados y 2) Potencialmente peligrosos (Tabla 1) ${ }^{19-20}$.

Desde principios del 2007 se inició en nuestra UCI un programa de farmacovigilancia, en conjunto con un equipo de químico-farmacéuticos. Este estudio se desarrolló en ese contexto y fruto de él. La UCI consta de 18 camas y, debido a la enorme cantidad de fármacos involucrados en estos pacientes, se decidió concentrarse en los de uso más frecuente, estos son los sedantes-analgésicos y antibióticos. Cada uno de los seleccionados fue confrontado con la literatura y luego con el programa de detección de interacciones Epocrates On Line $^{\circledR}$ (software de libre acceso $)^{19}$.

De la primera revisión efectuada se formaron dos grupos de fármacos, los contraindicados con una alta incidencia de producir el EA si la asociación se presentaba y los potenciales con una relación probable, si los fármacos se administraban en concomitancia. La revisión final en la confección de la tabla se basó en el programa Micromedex ${ }^{\circledR 20}$, que consiste en una base de datos actualizada con información al día de todos los medicamentos que se pueden usar en un servicio de salud, tanto en dosis, vías de administración, indicaciones y EA producidos, entre otras. Además, informa las notificaciones de EA desde que el medicamento empieza a comercializarse con las referencias específicas y renovadas constantemente. Dentro de sus herramientas se utilizaron los programas Drug REAX y Drug DEX, que indican de forma general los posibles problemas que pudiesen presentarse con la asociación de medicamentos ${ }^{20}$. Con la información resumida se diseñó la Tabla 1.

Los pacientes seleccionados de la población estudiada debían cumplir con los siguientes criterios de inclusión:

a) Tener al menos 3 días de estadía en UCI; b) Recibir algún(os) de los medicamento(s) estudiados con un mínimo de 3 días de terapia; c) Sin un límite de edad y considerando todas las patologías presentes.

Se evaluaron concomitantemente los datos epidemiológicos, el índice APACHE $\mathrm{II}^{21}$, el peso estimado, las patologías presentes, los días de estadía, los exámenes de laboratorio habituales y la mortalidad en la unidad.

Para la definición de cada uno de los EA se utilizó la guía "Common Terminology Criteria For Adverse Events" (CTCAE) versión 3.0, definida 
Tabla 1. Fármacos evaluados para las IEF y EA ${ }^{1}$

\begin{tabular}{|c|c|c|c|c|}
\hline $\begin{array}{l}\text { Fármaco } \\
\text { objeto }\end{array}$ & $\begin{array}{l}\text { Fármacos } \\
\text { contra- } \\
\text { indicados }\end{array}$ & $\begin{array}{l}\text { Eventos adversos } \\
\text { serios con fármacos } \\
\text { contraindicados }\end{array}$ & $\begin{array}{l}\text { Fármacos } \\
\text { potenciales }\end{array}$ & $\begin{array}{l}\text { Eventos adversos } \\
\text { comunes con fárma- } \\
\text { cos potenciales }\end{array}$ \\
\hline Amikacina & Cidofovir & $\begin{array}{l}\text { Falla renal aguda y } \\
\text { depresión del nivel } \\
\text { de conciencia }\end{array}$ & $\begin{array}{l}\text { Aciclovir, } \\
\text { Amfotericina B, } \\
\text { Ciclosporina, } \\
\text { Furosemida }\end{array}$ & $\begin{array}{l}\text { Falla renal aguda y } \\
\text { depresión del nivel de } \\
\text { conciencia }\end{array}$ \\
\hline $\begin{array}{l}\text { Amoxicilina/ } \\
\text { Clavulánico }\end{array}$ & Omeprazol & - & - & - \\
\hline Amfotericina B & Cidofovir & Falla renal aguda & $\begin{array}{l}\text { Aminoglicósidos oral } \\
\text { y parental, Digoxina }\end{array}$ & Falla renal aguda \\
\hline Carbapenémicos & Ganciclovir & $\begin{array}{l}\text { Reacción alérgica, } \\
\text { convulsiones y } \\
\text { taquicardia }\end{array}$ & $\begin{array}{l}\text { Micofenolato } \\
\text { Mofetil, Tramadol, } \\
\text { Ácido Valproico }\end{array}$ & Convulsiones \\
\hline Ceftriaxona & $\begin{array}{l}\text { Gluconato de } \\
\text { Calcio }\end{array}$ & $\begin{array}{l}\text { Precipitados de Calcio- } \\
\text { Ceftriaxona en vesícula } \\
\text { y pulmón }\end{array}$ & Cloruro de Calcio & $\begin{array}{l}\text { Precipitados de Calcio- } \\
\text { Ceftriaxona en vesícula y } \\
\text { pulmón }\end{array}$ \\
\hline Cotrimoxazol & $\begin{array}{l}\text { Amiodarona, } \\
\text { Metotrexato }\end{array}$ & Prolongación QT & $\begin{array}{l}\text { IECAs, Procaína } \\
\text { Fenitoina, Risperidona, } \\
\text { Quetiapina, Fluconazol }\end{array}$ & Hiperkalemia y ataxia \\
\hline Fentanilo & $\begin{array}{l}\text { Amiodarona, } \\
\text { Amlodipino, } \\
\text { Carvedilol, } \\
\text { Clonazepam }\end{array}$ & $\begin{array}{l}\text { Distress respiratorio, } \\
\text { bradicardia, convulsio- } \\
\text { nes, psicosis }\end{array}$ & $\begin{array}{l}\text { Midazolam, Propofol, } \\
\text { Lidocaína, Ácido } \\
\text { Valproico }\end{array}$ & $\begin{array}{l}\text { Distress respiratorio e } \\
\text { hipotensión }\end{array}$ \\
\hline Fluconazol & $\begin{array}{l}\text { Clonazepam, } \\
\text { Amlodipino, } \\
\text { Quetiapina }\end{array}$ & $\begin{array}{l}\text { Confusión, bradicardia e } \\
\text { hipotensión }\end{array}$ & $\begin{array}{l}\text { BZD, Claritromicina, } \\
\text { Risperidona }\end{array}$ & $\begin{array}{l}\text { Confusión, prolongación } \\
\text { QT, bradicardia }\end{array}$ \\
\hline Fluoroquinolonas & $\begin{array}{l}\text { Amiodarona, } \\
\text { Hidrocortisona }\end{array}$ & $\begin{array}{l}\text { Prolongación del interva- } \\
\text { lo QT, arritmias, ruptura } \\
\text { de tendón }\end{array}$ & $\begin{array}{l}\text { Cationes, Claritromicina, } \\
\text { Teicoplanina }\end{array}$ & $\begin{array}{l}\text { Arritmias cardíacas, } \\
\text { convulsiones }\end{array}$ \\
\hline Metronidazol & Amiodarona & $\begin{array}{l}\text { Prolongación del interva- } \\
\text { lo QT y arritmias }\end{array}$ & Fenitoina & Ataxia \\
\hline Propofol & Succinilcolina & Confusión & Lidocaína, Diazepam & Distress respiratorio \\
\hline Rifampicina & $\begin{array}{l}\text { Antifúngicos } \\
\text { Azólicos, } \\
\text { Atorvastatina }\end{array}$ & - & $\begin{array}{l}\text { Amlodipino, } \\
\text { Paracetamol, BZD }\end{array}$ & $\begin{array}{l}\text { Elevación de las } \\
\text { Transaminasas }\end{array}$ \\
\hline $\begin{array}{l}\text { Piperacilin/ } \\
\text { Tazobactam }\end{array}$ & Vecuronium & Distress respiratorio & Metotrexato & Falla renal aguda \\
\hline Vancomicina & Cidofovir & Falla renal aguda & $\begin{array}{l}\text { Aminoglicósidos oral y } \\
\text { parental, Amfotericina B }\end{array}$ & $\begin{array}{l}\text { Falla renal aguda y } \\
\text { depresión del nivel de } \\
\text { conciencia }\end{array}$ \\
\hline
\end{tabular}

'El diseño de la Tabla se llevó a cabo, mediante el uso de dos programas de detección de IEF, el Drug ReaX® de MICROMEDEX y Epocrates On Line Multicheck. El guión en la tabla representa que no existen fármacos y/o EA a evaluar para el estudio. 
por el Instituto Nacional del Cáncer de Estados Unidos $^{22}$.

Estadística: En el análisis estadístico se utilizó el programa SPSS (Statistical Package for the Social Sciences) 15.0 para Windows con los siguientes test: exacto de Fisher, riesgo relativo (RR) de la exposición y regresión logística multivariada en la obtención del odds ratio (OR). El tamaño muestral se determinó asumiendo una frecuencia de IEFs de $40 \%$, de acuerdo a lo antes descrito por la literatura investigada. La diferencia significativa entre ambos grupos se estableció con un nivel de seguridad de $95 \%$, un poder estadístico de $80 \%$ y un OR esperado mayor a 4 . En relación a los criterios señalados, el número de pacientes necesario fue de 34. La significancia mínima, para aceptar la hipótesis de relación, fue un valor p menor a 0,05 . Finalmente, en el caso del RR se construyó un intervalo de confianza al 95\% (IC 95\%) que determinó la verdadera relevancia de la exposición.

Tabla 2. Características generales del grupo seleccionado

\begin{tabular}{|c|c|c|c|c|}
\hline Variables pacientes & Promedio \pm DE & Mínimo y máximo & $\mathbf{n}$ & (\%) \\
\hline Edad & $58,63 \pm 16,3$ & $22-91$ & & - \\
\hline Peso & $76,77 \pm 11,056$ & $62-120$ & & - \\
\hline $\begin{array}{l}\text { Sexo } \\
\text { (F: femenino) } \\
\text { (M: masculino) }\end{array}$ & - & - & $\begin{array}{l}\text { F } 11 \\
\text { M } 24\end{array}$ & $\begin{array}{l}(31,4 \%) \\
(68,6 \%)\end{array}$ \\
\hline Apache & $15,54 \pm 5,49$ & $8-30$ & & - \\
\hline a) $\leq$ a 15 & - & - & 19 & $(54,2 \%)$ \\
\hline b) > a 15 & - & - & 16 & $(45,8 \%)$ \\
\hline \multicolumn{5}{|l|}{ Diagnóstico Ingreso } \\
\hline a) Sepsis & - & - & 12 & $(34,2 \%)$ \\
\hline Neumonía & - & - & 3 & $(8,5 \%)$ \\
\hline Abdominal & - & - & 6 & $(17,2 \%)$ \\
\hline Otros & - & - & & $3(8,5 \%)$ \\
\hline b) SDRA & - & - & 4 & $(11,6 \%)$ \\
\hline c) FRA & - & - & 3 & $(8,5 \%)$ \\
\hline d) PCR & - & - & 2 & $(5,7 \%)$ \\
\hline e) Otros & - & - & 14 & $(40 \%)$ \\
\hline Craniectomía & - & - & 2 & $(5,7 \%)$ \\
\hline Intoxicación & - & - & 1 & $(2,8 \%)$ \\
\hline AVE & - & - & 2 & $(5,7 \%)$ \\
\hline EPOC & - & - & 2 & $(5,7 \%)$ \\
\hline Meningitis & - & - & 2 & $(5,7 \%)$ \\
\hline Cardiopatía & - & - & 4 & $(11,6 \%)$ \\
\hline Fractura cadera & - & - & 1 & $(2,8 \%)$ \\
\hline f) Estadía UCl* & $23,35 \pm 18,66$ & $4-76$ & & \\
\hline g) Mortalidad & - & - & 3 & $(8,5 \%)$ \\
\hline
\end{tabular}

*Para el cálculo de la estadía en UCl, se excluyó un paciente por tener más de un año previo en la unidad. 


\section{Resultados}

Al final del período de estudio se totalizaron 35 pacientes que cumplieron con los criterios de inclusión definidos. La edad promedio fue de 58,63 $\pm 16,3$ años (mínimo 21 y máximo 91) con un peso estimado de 76,77 $\pm 11,05 \mathrm{~kg}$. La distribución por sexo fue $68,6 \%$ masculino y $31,4 \%$ femenino. El índice de Apache II fue de 15,54 \pm 5,49 (mínimo 8 y máximo 30 ).

Las causas de ingreso fueron: sepsis $34,2 \%$, síndrome de distress respiratorio en el adulto (SDRA) 11,6\%, falla renal aguda (FRA) $8,5 \%$, paro cardiorespiratorio (PCR) 5,7\% y otros, con $40 \%$, en el cual, la mayor porción correspondía a cardiopatía, con un 11,6\%. La estadía fue de 23,35 \pm 18,66 días (mínimo de 4 y máximo de 76) con un mortalidad promedio de 8,5\% (3 casos). Los datos mencionados se encuentran en la Tabla 2.

Se registraron un total de 76 IEF durante la totalidad de la investigación en los 35 pacientes. Para los fármacos clasificados como contraindicados, las IEF de mayor asociación fueron: fluoroquinolonas + hidrocortisona en 6 ocasiones, fentanilo + amiodarona en 5 y ceftriaxona + gluconato y/o cloruro de calcio en 2 oportunidades. En el caso de los fármacos clasificados como potencialmente peligrosos, las IEF de mayor asociación fueron: fentanilo + midazolam + propofol en 33 ocasiones, seguida de vancomicina + aminoglicósidos en 3 ocasiones y amfotericina $\mathrm{B}+$ vancomicina en otros 3. El total de la información se muestra en la Tabla 3.

Tabla 3. IEF encontradas

\begin{tabular}{|c|c|c|c|c|}
\hline Fármaco objeto & Fármacos contraindicados & $\mathbf{n}$ & Fármacos potenciales & $\mathbf{n}$ \\
\hline Amikacina & Cidofovir & 0 & $\begin{array}{l}\text { Aciclovir, Amfotericina B } \\
\text { Ciclosporina, Furosemida }\end{array}$ & 0 \\
\hline Amoxicilina/Clavulánico & Omeprazol [1] & 1 & - & 0 \\
\hline Amfotericina B & Cidofovir & 0 & Aminoglicósidos, Digoxina & 0 \\
\hline Carbapenémicos & Ganciclovir & 0 & $\begin{array}{l}\text { Micofenolato Mofetil } \\
\text { Tramadol, Ácido Valproico }\end{array}$ & 0 \\
\hline Ceftriaxona & Gluconato de Calcio [2] & 2 & Cloruro de Calcio & 0 \\
\hline Cotrimoxazol & $\begin{array}{l}\text { Amiodarona } \\
\text { Metotrexato }\end{array}$ & 0 & $\begin{array}{l}\text { IECAs Procaína } \\
\text { Fenitoina [3] Quetiapina } \\
\text { Risperidona Fluconazol }\end{array}$ & 3 \\
\hline Fentanilo & $\begin{array}{l}\text { Amiodarona [5] } \\
\text { Amlodipino [3] } \\
\text { Carvedilol [1] } \\
\text { Clonazepam [1] }\end{array}$ & 10 & $\begin{array}{l}\text { Midazolam [23] } \\
\text { Propofol [10] } \\
\text { Lidocaína [2] } \\
\text { Ácido Valproico [1] }\end{array}$ & 36 \\
\hline Fluconazol & $\begin{array}{l}\text { Clonazepam } \\
\text { Amlodipino [1] Quetiapina }\end{array}$ & 1 & $\begin{array}{l}\text { Benzodiazepinas [3] } \\
\text { Claritromicina Risperidona }\end{array}$ & 3 \\
\hline Fluoroquinolonas & $\begin{array}{l}\text { Amiodarona [1] } \\
\text { Hidrocortisona [6] }\end{array}$ & 7 & $\begin{array}{l}\text { Cationes } \\
\text { Claritromicina Teicoplanina }\end{array}$ & 0 \\
\hline Metronidazol & Amiodarona & 0 & Fenitoina [3] & 3 \\
\hline Propofol & Succinilcolina & 0 & $\begin{array}{l}\text { Diazepam } \\
\text { Lidocaína [2] }\end{array}$ & 2 \\
\hline Rifampicina & $\begin{array}{l}\text { Antifúngicos Azólicos [1] } \\
\text { Atorvastatina }\end{array}$ & 1 & $\begin{array}{l}\text { Amlodipino Paracetamol } \\
\text { BZD [1] }\end{array}$ & 1 \\
\hline Piperacilin/Tazobactam & Vecuronium & 0 & Metotrexato & 0 \\
\hline Vancomicina & Cidofovir & 0 & $\begin{array}{l}\text { Aminoglicósidos [5] } \\
\text { Amfotericina B [1] }\end{array}$ & 6 \\
\hline
\end{tabular}

[]: Corresponde a las veces en la que asociación estuvo presente en la terapia. n: Cantidad total de asociaciones entre los 14 fármacos y los grupos contraindicados y potenciales. 
Tabla 4. EAF encontrados

\begin{tabular}{|lc|}
\hline $\begin{array}{l}\text { Evento adverso a fármacos } \\
\text { estudiados }\end{array}$ & Cantidad \\
\hline Reacción alérgica & 2 \\
\hline Arritmias cardíacas & 4 \\
$\begin{array}{l}\text { Bradicardia (frecuencia cardíaca bajo } 60 \\
\text { latidos por minuto) }\end{array}$ & 21 \\
\hline Convulsiones & 1 \\
\hline Psicosis & 0 \\
\hline Distress respiratorio (SDRA) & 6 \\
\hline Confusión & 1 \\
\hline Elevación transaminasas & 2 \\
\hline Hemorragia & 3 \\
Hipotensión & 6 \\
\hline Hiperkalemia & 0 \\
Falla renal aguda (FRA) & 7 \\
\hline Depresión del nivel de conciencia & 0 \\
Prolongación del intervalo QT & 2 \\
\hline $\begin{array}{l}\text { Taquicardia (frecuencia cardíaca mayor a } \\
\text { 100 latidos por minuto) }\end{array}$ & 0 \\
Ataxia & 0 \\
\hline fuerza) & 5 \\
\hline Retención urinaria & \\
\hline
\end{tabular}

Para los EA el total de casos llegó a 60, con una distribución marcada para la bradicardia (21 oportunidades), la que siguió en número fue la FRA en 7 ocasiones, la hipotensión y el SDRA, ambos con 6 casos y finalmente la retención urinaria con 5 . La frecuencia de los EA encontrados esta descrita en la Tabla 4.

En la determinación de la posible relación entre los EA producidos y las IEF estudiadas se construyen las Tablas 5 y 6 , las que resumen cuáles son los fármacos evaluados con sus respectivos EA. En cada tabla se aplican los test estadísticos anteriormente enunciados.

De acuerdo al test exacto de Fisher, las IEF asociadas a la producción de EA se encuentran en la Tabla 5. En la Tabla 6 se resumen los valores estadísticos para la posible relación entre IEF y EA. Las IEF más importantes fueron: fentanilo +
Tabla 5. Probables interacciones asociadas a los EAF detectados

\begin{tabular}{|c|c|}
\hline $\begin{array}{l}\text { Evento adverso a } \\
\text { fármacos estudiados }\end{array}$ & $\begin{array}{l}\text { Interacción } \\
\text { probable }\end{array}$ \\
\hline Reacción alérgica & Piperacilin/Tazobactam \\
\hline Arritmias cardíacas & $\begin{array}{l}\text { Fentanilo- } \\
\text { Amlodipino }\end{array}$ \\
\hline $\begin{array}{l}\text { Bradicardia } \\
\text { (Frecuencia cardíaca } \\
<60 \text { latidos/min) }\end{array}$ & $\begin{array}{l}\text { Fentanilo- } \\
\text { Midazolam- } \\
\text { Propofol }\end{array}$ \\
\hline SDRA & $\begin{array}{l}\text { Fentanilo- } \\
\text { Midazolam- } \\
\text { Propofol }\end{array}$ \\
\hline Confusión & $\begin{array}{l}\text { Midazolam- } \\
\text { Fluconazol- } \\
\text { Amlodipino }\end{array}$ \\
\hline Hipotensión & $\begin{array}{l}\text { Fentanilo- } \\
\text { Midazolam- } \\
\text { Propofol }\end{array}$ \\
\hline Falla renal aguda & $\begin{array}{l}\text { Vancomicina- } \\
\text { Amikacina, } \\
\text { Amfotericina B- } \\
\text { Vancomicina }\end{array}$ \\
\hline $\begin{array}{l}\text { Prolongación del } \\
\text { intervalo QT }\end{array}$ & $\begin{array}{l}\text { Amiodarona- } \\
\text { Moxifloxacino }\end{array}$ \\
\hline Retención urinaria & $\begin{array}{l}\text { Fentanilo- } \\
\text { Propofol }\end{array}$ \\
\hline
\end{tabular}

midazolam + propofol asociadas con la producción de bradicardia e hipotensión con un RR de 10,4 (IC 95\% 1,59-68) y 5 (IC 95\% 1,08-23,1), respectivamente. El OR calculado para bradicardia e hipotensión según la regresión logística multivariada fue de 125,2 (IC 95\% 1,8-1.224) y 12,6 (IC 95\% 1,2-124), respectivamente.

Otra IEF importante fue vancomicina + amfotericina B y amikacina + vancomicina asociada con la producción de FRA, con un RR y OR de 6,4 (IC 95\% 1,9-21,6) y 10,8 (IC 95\% 1,2-76). Las diferencias obtenidas entre las cantidades totales de EA producidos referidos en las Tablas 4 y 6 , se deben a que en la segunda, sólo se evaluaron los casos de EA cuando las IEF estuvieron presentes en la terapia.

La prolongación del intervalo QT, retención urinaria, arritmias y SDRA no mostraron relación 
Tabla 6. Valores estadísticos para la relación entre IEF y EA

\begin{tabular}{|c|c|c|c|c|c|}
\hline Interacción entre fármacos & $\begin{array}{c}\text { EAF } \\
\text { evaluados }\end{array}$ & Cantidad & Valor $\mathbf{p}$ & $\mathbf{R R}$ & OR \\
\hline Fentanilo-Midazolam-Propofol & Bradicardia & 20 & $p<0,001$ & $10,4(1,59-68)$ & $125,2(1,8-1224)$ \\
\hline Fentanilo-Midazolam-Propofol & Hipotensión & 4 & 0,043 & $5 \quad(1,08-23,1)$ & $12,6(1,2-124)$ \\
\hline $\begin{array}{l}\text { Vancomicina-Amikacina y } \\
\text { Vancomicina-Amfotericina B }\end{array}$ & FRA & 4 & 0,009 & $6,4(1,9-21,6)$ & $10,8(1,2-76)$ \\
\hline Moxifloxacino-Amiodarona & $\begin{array}{l}\text { Prolongación } \\
\text { QT }\end{array}$ & 1 & 0,057 & $34 \quad(4,9-234)$ & - \\
\hline Propofol-Fentanilo & $\begin{array}{l}\text { Retención } \\
\text { urinaria }\end{array}$ & 5 & $p<0,001$ & - & - \\
\hline Fentanilo-Amlodipino & Arritmias & 1 & 0,114 & $11,3(3,8-33,4)$ & - \\
\hline Fentanilo-Midazolam-Propofol & SDRA & 2 & 0,344 & $2 \quad(0,45-8,8)$ & $2,677(0,56-7,9)$ \\
\hline
\end{tabular}

Tabla 7. Regresión logística multivariada

\begin{tabular}{|c|c|c|c|c|c|c|}
\hline \multirow{3}{*}{$\begin{array}{l}\text { Odds ratio regresión logística multivariada } \\
\text { Edad }\end{array}$} & \multicolumn{6}{|c|}{ Eventos adversos con significancia (OR) } \\
\hline & \multicolumn{2}{|c|}{ Bradicardia* } & \multicolumn{2}{|c|}{ Hipotensión* } & \multirow{2}{*}{\multicolumn{2}{|c|}{$\begin{array}{c}\mathbf{F R A}^{*} \\
1,003(0,7-2,1)\end{array}$}} \\
\hline & 1 & $(0,6-2)$ & 0,97 & $(0,7-2,3)$ & & \\
\hline Peso & 0,996 & $6(0,9-1,3)$ & 1,125 & $5(0,9-1,3)$ & 1,2 & $(0,8-1,5)$ \\
\hline Apache II de ingreso & 0,959 & $9(0,7-1,1)$ & 1,05 & $(0,75-2,6)$ & 1,038 & $8(0,9-1,0)$ \\
\hline Presencia de cardiopatía & 2,508 & $8(0,87-4)$ & 2,8 & $(0,9-5)$ & 1,84 & $(0,9-1,2)$ \\
\hline IRA (creatinina de ingreso mayor a 1,3 mg/dL) & 1,57 & $(0,9-3,6)$ & 1,2 & $(0,9-1,9)$ & 3,1 & $(0,9-1,07)$ \\
\hline Uso de medios de contraste & \multicolumn{2}{|r|}{ N.A. } & \multicolumn{2}{|r|}{ N.A. } & \multicolumn{2}{|r|}{ N.A. } \\
\hline AINES & \multicolumn{2}{|r|}{ N.A. } & \multicolumn{2}{|r|}{ N.A. } & \multicolumn{2}{|r|}{ N.A. } \\
\hline Diabetes mellitus & \multicolumn{2}{|r|}{ N.A. } & \multicolumn{2}{|r|}{ N.A. } & & N.A. \\
\hline
\end{tabular}

*El valor del intervalo de confianza al 95\% para estas variables, atraviesa el valor 1, determinando que ninguna posee relación con la interacción y el evento adverso. N.A.: No Aplica.

estadísticamente significativa. Para la retención urinaria el tamaño muestral no permitió el cálculo del RR y del OR, ya que no hubo IEF para el EA.

Para identificar otros factores de riesgo que pudieran estar implicados en la producción del EA, se realizó un análisis multivariado donde se incluyó edad, peso estimado, índice APACHE II de ingreso, presencia de cardiopatía, insuficiencia renal aguda (IRA), diabetes mellitus, uso de medios de contraste y uso de antiinflamatorios no esteroidales (AINES); no demostrándose asociación con ellos (Tabla 7).

\section{Discusión}

Según nuestro conocimiento, este sería el primer estudio de IEF en el contexto de UCIs en nuestro país. Llama la atención que dada la frecuencia descrita de hasta $23 \%$ de eventos adversos clínicamente significativos atribuibles a las IEF, existe poca conciencia en nuestro medio de su real importancia.

Las UCIs concentran quizás la mayor polifarmacia entre los pacientes hospitalizados, de modo que este estudio cumple el objetivo de describir su presencia en nuestra Unidad. 
Se reunió la información de la totalidad de pacientes que ingresaban a la unidad, siendo 35 pacientes los estudiados, suficientes según el cálculo de tamaño muestral descrito. Para todos los fármacos estudiados se utilizó como sistema de revisión el programa Micromedex ${ }^{\circledR}$, con sus herramientas Drug REAX y Drug DEX que analizó el total de las 14 asociaciones con los dos grupos definidos como contraindicados y potencialmente peligrosos.

Se registraron un total de 76 IEF durante la investigación en los 35 pacientes seleccionados. En los fármacos contraindicados, las IEF de mayor asociación fueron: fluoroquinolonas + hidrocortisona en 6 ocasiones, fentanilo + amiodarona en 5 y ceftriaxona + gluconato y/o cloruro de calcio en 2 oportunidades. En el caso de los fármacos potencialmente contraindicados, la interacción para fentanilo + midazolam + propofol se asoció en 33 ocasiones, seguida de vancomicina + aminoglicósidos y/o amfotericina B en 6 .

Para la mayoría de los casos que tenían concomitantemente la presencia de la asociación fentanilo + midazolam + propofol se presentaron los siguientes EA, que encabeza la bradicardia con $35 \%$ e hipotensión y SDRA con 10\%. La marcada disminución de la frecuencia cardíaca por la presencia de la IEF fue el resultado más visto. El EA se producía cuando la IEF estaba en la terapia de los pacientes, pero a la hora de determinar que cada paciente sufriera este resultado, se debían eliminar otras variables. Dentro de ellas estaban edad, peso y APACHE II, que evaluaron si los pacientes tenían predisposición a la producción del EA por alguna característica individual. La variable presencia de cardiopatía previa en el paciente no mostró significancia estadística por regresión logística multinomial, al igual que la edad, que tanto para pacientes más jóvenes como mayores se presentó, el peso que se asoció a la dosis administrada no mostró mayor valor y el APACHE II de ingreso que atribuía la gravedad propia del ingresado a la unidad no difirió de los anteriores resultados con OR cercano a 1. El RR calculado de la IEF en producir el EA fue de 10,4 en bradicardia y de 5 en hipotensión concluyendo que sólo debían estar presentes los fármacos, sin importar otras variables con un OR de 125,2 y 12,6, respectivamente.

Para la asociación entre vancomicina + amikacina y vancomicina + amfotericina $B$, la cantidad de episodios de FRA llegó a 7 casos, de los cuales
3 se eliminaron de todo análisis, pues formaban parte de las patologías de ingreso a la unidad. Con los 4 casos restantes se evaluaron ambas IEF, observando un aumento gradual en los valores de creatinina comenzado el tratamiento. Los valores de riesgo relativo y odds ratio fueron 6,4 y 8 respectivamente, sin importar la edad, el peso, el APACHE II, IRA y uso de medios de contraste, calculado por regresión logística multinomial. Es importante señalar que las dosis utilizadas fueron dosis habituales (datos no mostrados) y en ningún momento fueron dosis tóxicas, lo que apoya la tesis de la etiología de los EA atribuibles a las IEF ya descritas.

No podemos establecer la prevalencia real de las IEF y los EA producidos, ya que no es el objetivo del presente estudio y el diseño no lo permite. Sin embargo, pretendemos sensibilizar al respecto $y$ abrir el camino para nuevos estudios multicéntricos y con mayor poder que puedan resolver estas preguntas.

En suma, podemos concluir que las IEF y los EA asociados a ellos son un problema frecuente, convirtiéndose en un tema relevante en nuestra UCI y probablemente en otras del país.

Las IEF que se asociaron con riesgo en la producción de EAs son: fentanilo + propofol + midazolam para bradicardia e hipotensión; amfotericina B + vancomicina y vancomicina + amikacina para FRA. En el manejo tanto de las IEF y de los EA producidos, la farmacovigilancia se convierte en una herramienta eficaz para su detección y prevención.

Finalmente, el trabajo mutuo y coordinado de médicos y químico-farmacéuticos y todo el equipo de salud, hace posible la pesquisa y comprensión de nuevos problemas, antes no visualizados en el manejo diario de nuestros pacientes. De esta manera podemos optimizar las terapias y buscar mejores soluciones para ellos.

\section{Referencias}

1. Organización Mundial de la Salud. WHO Department of Essential Drugs and Medicines. The Uppsala Monitoring Centre. The importance of pharmacovigilance, safety monitoring of medicinal products. Ginebra (2001). Disponible en: http://www.who.int/medicinedocs/pdf/ s2288e/s2288e.pdf

2. International drug monitoring: the role of national centers. Organización Mundial de la Salud. Génova; 1972. 
(Technical Reports Series, 498). Disponible en: http:// www.who-umc.org/DynPage.aspx?id=13136

3. Wolff RM. Consecuencias adversas inesperadas durante el uso de antimicrobianos: Cuándo el tratamiento puede ser peligroso para la salud. Rev Chil Infect 2002; 19 Suplemento 1.

4. U.S. Food and Drug Administration. Drug Development and Drug Interactions (2006). www.fda.com

5. NCCMERP Taxonomy of medication errors. National Coordinating Council for Medication Error Reporting and Prevention. (2001). Disponible en: http://www.nccmerp. org/pdf/taxo2001-07-31.pdf.

6. Morales M, Ruiz I, Morgado C, González X. Farmacovigilancia en Chile y el mundo. Rev Chil Infect 2002; 19 Suplemento 1 .

7. Kuhlmann J, Muck W. Clinical-pharmacological strategies to assess drug interaction potential during drug development. Drug Saf 2001; 24: 715-25.

8. Johnson JA, Bootman JL. Drug-related morbidity and mortality. Arch Inter Med 1995; 155: 1949-56.

9. Grymonpre RE, Mitenko PA, Sitar DS. Drug-associated hospital admissions in older medical patients. J Am Geriatr Soc 1988; 36: 1092-8.

10. White TJ, Arakelian A, Rho JP. Counting the costs of drug-related adverse events. Pharmacoeconomics 1995; 15: 445-58.

11. Abarca J, Malone DC, Armstrong EP. Identification of Serious Drug-Drug Interactions: Results of the Partnership to Prevent Drug-Drug Interactions. J Am Pharm Assoc 2004; 44: 136-41.
12. Bergk V, Gasse C, Rothenbacher D, Loew M, Brenner H, Haefeli WE. Drug interactions in primary care: impact of a new algorithm on risk determination. Clin Pharmacol Ther 2004; 76: 85-96.

13. Hazlet TK, Lee TA, Hansten PD. Performance of community pharmacy drug interaction software. J Am Pharm Assoc (Wash DC) 2002; 41: 200-4.

14. Barrons R. Evaluation of personal digital assistant software for drug interactions. Am J Health Syst Pharm 2004; 61: 380-5.

15. Gandhi TK, Weingart SN, Borus J, Seger AC, Peterson J, Burdick E, et al. Adverse Drug Events in Ambulatory Care. N Engl J Med 2003; 348: 1556-64.

16. Gurwitz JH, Field TS, Harrold LR, et al. Incidence and preventability of adverse drug events among older persons in the ambulatory setting. JAMA 2003; 289: 1107 16.

17. Tierney WM. Adverse outpatient drug events: a problem and an opportunity. N Engl J Med 2003; 348: 1587-9.

18. Classen D. Medication safety: moving from illusion to reality. JAMA 2003; 289: 1154-6.

19. Sistema Epocrates On Line ${ }^{\circledR}$ www.epocrates.com

20. Micromedex ${ }^{\circledR}$ Healthcare Series Vol. 143 expires 3/2010. (C) 1974-2010 Thomson Reuters. All rights reserved.

21. Knaus WA, Draper EA, Wagner DP, Zimmerman JE. APACHE II: a severity of disease classification system. Crit Care Med 1985; 13: 818-29.

22. http://ctep.cancer.gov/reporting/ctc.html Cancer Therapy Evaluation Program. National Cancer Institute. Reporting guidelines. Agosto 2006. 